\title{
Amelioration of Obesity, Glucose Intolerance, and Oxidative Stress in High-Fat Diet and Low-Dose Streptozotocin-Induced Diabetic Rats by Combination Consisting of "Curcumin with Piperine and Quercetin"
}

\author{
Ginpreet Kaur and Meena C \\ SVKM's Narsee Monjee Institute of Management Studies (NMIMS), School of Pharmacy \& Technology Management, \\ Vile Parle (W), Mumbai 400056, India \\ Correspondence should be addressed to Ginpreet Kaur, ginpreet.aneja@gmail.com
}

Received 30 November 2011; Accepted 21 December 2011

Academic Editors: T. Kumai and M. A. Prendergast

Copyright ( 2012 G. Kaur and M. C. This is an open access article distributed under the Creative Commons Attribution License, which permits unrestricted use, distribution, and reproduction in any medium, provided the original work is properly cited.

\begin{abstract}
Curcumin is an important nutraceutical that has enormous potential for a variety of diseases, but the medicinal properties of curcumin cannot be utilized due to its low in vivo bioavailability. Therefore, in view of the foregoing, there is an extensive need for combinatorial extract "curcumin with piperine and quercetin" which may enhance bioavailability of oral curcumin by inhibiting the enzymes responsible for the metabolism of curcumin. Thus, the present study investigated the effect of combinatorial extract of curcumin on obesity, glucose intolerance, and oxidative stress in high fat diet and low-dose streptozotocin-induced rats. Oral administration of combinatorial extract for 28 days significantly $(P<0.05)$ reduced PGL $(64.84 \%)$, PTG (88.94\%), LDL (26.38\%) and PTC $(50.23 \%)$ levels, respectively and improved glucose tolerance $(P<0.05)$ significantly to exogenously administered glucose $(2 \mathrm{~g} / \mathrm{kg})$ at 60,90 , and $120 \mathrm{~min}$ interval on OGTT. The results for antioxidant potential indicate that at $100 \mathrm{mg} / \mathrm{kg}$ dose of combinatorial extract of curcumin significantly prevented the high-fat diet and low-dose streptozotocin-induced changes in the oxidative stress parameters $(P<0.01)$ which supports popular medicinal uses of this combinatorial extract as antihyperglycemic and hypolipidemic and is likely to bring this promising natural product to the forefront of therapeutic agents in the in the treatment of "metabolic syndrome".
\end{abstract}

\section{Introduction}

Metabolic syndrome, a concurrence of disorders including obesity, insulin resistance, dyslipidemia, and hypertension, has gained importance because of its association with subsequent development of cardiovascular disease and type 2 diabetes [1]. In contemporary times, the metabolic disorder is becoming an alarming concern for the developed as well as the developing world. The prevalence of metabolic syndrome is increasing worldwide and is a growing threat to global health, since it accounts for $6-7 \%$ of all-cause mortality. People with metabolic syndrome are twice as likely to die from heart attack or stroke compared with people without the syndrome and has a five fold greater risk of developing type 2 diabetes $[2,3]$ It is one of the biggest health concerns of our time as it affects more than one-third of the population, and it will continue to plaque the world at an alarming rate. Diet, exercise, and nutritional supplementation play a key role for maintaining a healthy lifestyle and well being of an individual, especially related to cardiovascular diseases, despite remarkable advances in medicine and pharmaceutical drug development. Thus, the current scenario possesses a need to address and focus our research on nutraceuticals and its role in metabolic syndrome. Curcumin, an important nutraceutical obtained from the root of the plant Curcuma longa Linn (family Zingiberaceae), is a perennial herb that has immense medicinal properties and is a nontoxic, highly promising natural compound having a wide spectrum of biological functions such as antiinflammatory [4], hepato- and nephro-protective $[5,6]$, 
antioxidant, [7-9] antimicrobial, [10] anticarcinogenic [11], and thrombosis-suppressing properties [12, 13]. Although Curcuma longa has been investigated for its various medicinal properties, detailed studies on its antidiabetic, antioxidant potential, and lipid peroxidation level in diabetic rats are still lacking as the medicinal properties of curcumin cannot be utilized due to its limited aqueous solubility and degradation at alkaline $\mathrm{pH}$ which restricts its bioavailability. Therefore, in view of the foregoing, there is an extensive need for such combination of curcumin with piperine and quercetin as piperine is an alkaloid found naturally in plants as Piper nigrum L, commonly known as black pepper is established as a bioavailability enhancer of drugs. It is an inhibitor of glucuronidation metabolism hence increases bioavailability (serum concentration) of oral curcumin [14], and if a second bioavailability enhancer such as quercetin is added, an increase in absorption is possible to a greater extent as it aids in the inhibition of metabolic conversion of curcumin. Thus, the present study was envisaged to determine the effect of combinatorial extract "curcumin with piperine and quercetin" on glucose intolerance and oxidative stress in high-fat diet and low-dose streptozotocin-induced rats. In addition the antiobesity effect of combinatorial extract of curcumin on diet-induced changes in body weight, plasma glucose, triglyceride cholesterol, and low-density lipoprotein (LDL) were also determined.

\section{Materials and Methods}

2.1. Animals. Albino female wistar rats ( 11 weeks old) were used in the present investigation. The animals were maintained in polypropylene cage in the Departmental Animal House Facility, with $12 \mathrm{~h}$ light: $12 \mathrm{~h}$ dark cycle. All the animals were kept under laboratory condition (temperature 25 $\pm 2^{\circ} \mathrm{C}$; relative humidity $75 \% \pm 5 \%$ ) for an acclimatization period of 7 days before carrying out the experiments. During the experiments animals were provided with standard rodent pellet diet (Amrut laboratory animal feed, Maharashtra), and filtered water was provided ad libitum. The experiment was approved by Animal ethical committee, Department of Pharmacology, School of Pharmacy \& Technology Management, Narsee Monjee Institute of Management and Studies, Mumbai, and conducted according to the guidelines of Committee for the Purpose of Control and Supervision of Experimentation on Animals, India.

2.2. Drugs. Streptozotocin was purchased from Sigma chemicals, USA. Glucose Oxidase kit was purchased from Transasia Biomedicals Limited, Mumbai. All other chemicals were of analytical grade and obtained from local suppliers.

2.3. Collection and Authentication of Plant Material. The rhizomes of Curcuma longa Linn, fruits of Piper nigrum and red onion of Allium cepa were procured in the months of March-June from the local market of Mumbai, India. Specimen samples were authenticated by Department of Raw and Crude drug material, National Institute of Science Communication and Information Resource (NISCAIR), New
Delhi. The voucher specimens were deposited in department for future reference.

2.4. Extraction and Isolation of Active Constituents. Extraction and isolation of curcuminoid, piperine, and quercetin from Curcuma longa Linn, piper nigrum and Allium cepa was done using petroleum ether, chloroform, and ethanol as a solvent. The identity of the compound was confirmed by its comparison with reference standard using thin-layer chromatography (TLC). TLC was developed by using mobile phase, as chloroform:methanol $(9: 1)$ for curcumin, toluene: ethyl acetate $(7: 3)$ for piperine, and chloroform: methanol:toluene $(7: 3: 1)$ for quercetin. The $R_{f}$ value was found to be similar with that of the standard.

\subsection{Preparation of Combinatorial Extract of Curcumin.} Combinatorial extract was prepared by suspending curcumin : piperine : quercetin in a ratio $(94: 1: 5)$ in $5 \%$ Gum Acacia and $0.5 \%$ tween 80 . Required quantity of gum acacia was weighed and taken in a mortar. It was triturated with very little quantity of tween 80 to form slurry. Accurately weighed quantity of curcumin, piperine, and quercetin was added to the slurry and trituration continued. The slurry was transferred to a measuring cylinder, and the volume was made up with water. The suspension was transferred to a vial and shaken thoroughly.

2.6. Acute Toxicity Study. The acute toxicity of combination consisting of curcumin with piperine and quercetin (CPQ) was evaluated in mice using the OECD guidelines 423 [15]. Swiss albino mice were randomly divided into three groups, each containing six animals. The combination consisting of CPQ was administered orally at doses of 500, 1000, and $2,000 \mathrm{mg} / \mathrm{kg}$ of body weight. Distilled water was administered to control group. The general behavior of the mice was continuously monitored for $1 \mathrm{~h}$ after dosing, periodically during the first $24 \mathrm{~h}$ with special attention given during the first $4 \mathrm{~h}$, and daily thereafter, for a total of 14 days. Changes in the normal activity of mice and their body weights were monitored and the time at which signs of toxicity or death appeared was recorded.

2.7. Treatment Schedule and High-Fat Diet and Low-Dose Streptozotocin-Induced Metabolic Abnormalities. After acclimation in the laboratory for one week, the rats were divided into seven groups (weighing $100-150 \mathrm{~g}$ ) to receive the following treatments: group 1 received drug vehicle only (5\% Gum Acacia and 0.5\% tween 80), (normal control); group2 received single intraperitoneal injection of freshly prepared solution of streptozocin (STZ) in a dose of $30 \mathrm{mg} / \mathrm{kg}$ body weight, (STZ control); group 3 received high-fat diet, (HFD control); group 4 received high-fat diet and STZ, (HFD + STZ 30); group 5 received high-fat diet with STZ and treated with $100 \mathrm{mg} / \mathrm{kg}$ curcumin alone, (CE-100 mg/kg + HFD + STZ 30); group 6 received high-fat diet with STZ and treated with combination consisting of curcumin with piperine and quercetin at the dose of $100 \mathrm{mg} / \mathrm{kg},(\mathrm{CBE}+\mathrm{HFD}+\mathrm{STZ} 30$ ); group 7 received high-fat diet with STZ, and treated with 
$50 \mathrm{mg} / \mathrm{kg}$ of combination consisting of curcumin with piperine and quercetin, (CBE + HFD + STZ 30). The HFD was formulated twice a week and stored at $4{ }^{\circ} \mathrm{C}$ in the refrigerator. The extracts were solubilized in 5\% Gum Acacia and $0.5 \%$ tween 80 and were administered by oral gavage from 30 to 60 days. On day 30, the animals were injected with single intraperitoneal injection of freshly prepared solution of STZ in a dose of $30 \mathrm{mg} / \mathrm{kg}$ body weight dissolved in citrate buffer ( $\mathrm{pH} 4.5)$. The rats were kept on the above treatment and were continued to be fed with the high-fat diet until day 60. A separate group of animals were fed with normal diet $(20 \mathrm{~g} /$ day/rat $)$ and did not receive streptozocin injection (nondiabetic control). During this period body weight, feed and water intake of the animals were also measured initially as well as estimated at the end of 8 weeks. At the end of experimental period, all the animals were anesthetized and blood samples were withdrawn from retro-orbital route, using heparin as anticoagulant. No significant effect of ether anaesthesia was observed on the plasma parameters measured.

2.8. Determination of Plasma Metabolic Parameters. Plasma samples obtained from the rats were used to estimate the metabolic parameters. Plasma glucose (PGL), triglyceride (PTG), cholesterol (PTC), high-density lipoprotein (HDL), and low-density lipoprotein (LDL) were analyzed using semiautomated bioanalyser using commercially available kits.

2.9. Oral Glucose Tolerance Test. At the end of 8 weeks, all the rats from each group were fasted for $16 \mathrm{~h}$ before being subjected to an oral glucose tolerance test (OGTT) by giving oral glucose load- $2 \mathrm{~g} / \mathrm{kg}$ p.o. [16]. The blood was withdrawn from the retro orbital sinus at $0,15,30,60,90$, and $120 \mathrm{~min}$ after the glucose load from all the animals for the estimation of glucose. The fasting blood glucose levels were estimated by the procedure as mentioned by the manufacturer of the kit (Erba Diagnostics, Germany).

\subsection{Assessment of Oxidative Stress Parameters}

2.10.1. Preparation of Erythrocyte Lysate. Blood was collected in a vacutainer precoated with anticoagulant. It was centrifuged at $3000 \mathrm{rpm}$ for $15 \mathrm{~min}$ in a cooling centrifuge $\left(4^{\circ} \mathrm{C}\right)$. Plasma was separated, and the packed cell volume (PCV) was washed three times with $0.9 \%$ sodium chloride (normal saline). To $0.5 \mathrm{~mL}$ of $5 \%$ suspension of packed cell volume in saline, $0.5 \mathrm{~mL}$ of ice cold distilled water was added and kept aside for $5 \mathrm{~min}$ at $4^{\circ} \mathrm{C}$. To this, $0.4 \mathrm{~mL}$ mixture of chloroform: ethanol $(3: 5)$ was added. The contents were mixed well and centrifuged at $3000 \mathrm{rpm}$ for $20 \mathrm{~min}$ at $4^{\circ} \mathrm{C}$. The water layer separated was used as erythrocyte lysate.

2.10.2. Estimation of Catalase. Catalase activity was assayed by the method of Aebi, (1974) [17]. Briefly, the assay mixture consisted of $1.95 \mathrm{~mL}$ phosphate buffer: $0.05 \mathrm{M}$ (pH 7.0) containing $5 \mathrm{mM}$ EDTA, $1 \mathrm{~mL}$ of hydrogen peroxide $(30 \mathrm{mM}$ solution) and $0.05 \mathrm{~mL}$ erythrocyte lysate in a final volume of $3.0 \mathrm{~mL}$. Changes in absorbance were recorded $240 \mathrm{~nm}$. One unit of CAT is defined as the number of $\mu$ mole of $\mathrm{H}_{2} \mathrm{O}_{2}$ decomposed per min at $25^{\circ} \mathrm{C}$.

2.10.3. Estimation of Reduced Glutathione. Glutathione activity was assayed by the method of Beutler et al., 1963 [18]. Briefly, the assay mixture consisted of $0.4 \mathrm{~mL}$ of erythrocyte lysate in $1.5 \mathrm{~mL}$ Tris $\mathrm{HCl}$ buffer $(\mathrm{pH} 8.00)$ and 0.25 of $5,5^{\prime}$ dithiobis-(2-nitrobenzoic acid) DTNB was added. Intensity of the colour was measured at $412 \mathrm{~nm}$ against blank. Glutathione in blood was then calculated as $\mu$ moles per gram of hemoglobin.

2.10.4. Estimation of Superoxide Dismutase. Glutathione activity was assayed by the method of Marklund, (1984) [19]. Briefly, the assay mixture consisted of $0.1 \mathrm{~mL}$ of erythrocyte lysate in $2.8 \mathrm{~mL}$ of Tris $\mathrm{HCl}$ buffer $(50 \mathrm{mM})$ and $0.1 \mathrm{~mL}$ of Pyrogallol $(30 \mathrm{mM})$. The rate of autoxidation was observed by measuring change in optical density at $420 \mathrm{~nm}$ for $2 \mathrm{~min}$ was recorded. One unit of SOD is described as the amount of enzyme required to cause $50 \%$ inhibition of pyrogallol auto oxidation $/ 3 \mathrm{~mL}$ of assay mixture.

2.10.5. Histopathology Analysis. Liver, kidney, spleen, and pancreas were fixed immediately in $10 \%$ formalin for routine histopathological examination. The tissues were embedded in paraffin, and then sectioned, stained with haematoxylin and eosin and were examined under light microscope. Histopathological evaluations were performed by pathologist. Photomicrographs of the microscopical sections were taken with the help of Motic photomicroscope (Canada) provided with Motic Images plus 2.0 software.

2.11. Statistical Analysis. The differences among experimental and control groups were determined using the Graph Pad INSTAT 3.0 software for Windows. Comparisons among different groups were performed by analysis of variance using ANOVA test. Significant difference between control and experimental groups were assessed by student's $t$-test. All data are expressed as mean \pm standard error of mean (S.E.M.); $P$ values less than 0.05 were considered to be significant.

\section{Results}

The present study demonstrates that the rats fed with highfat diet combined with low-dose streptozotocin resulted in significant elevation of plasma glucose as evident from elevated glycemic levels at 30,60, 90, and $120 \mathrm{~min}$ after glucose challenge, compared with the vehicle-treated normal pellet diet (NPD) control group $(P<0.05)$ and exhibited the characteristic features of obesity and glucose intolerance namely increased body weight, hypertriglyceridemia, hypercholesterolemia and impaired glucose tolerance compared with NPD fed control groups.

\subsection{Effect of CPQ on Biochemical Parameters in High-Fat Diet and Low-Dose Streptozotocin-Induced Diabetic Rats.} The plasma glucose, triglyceride, cholesterol, LDL levels in 


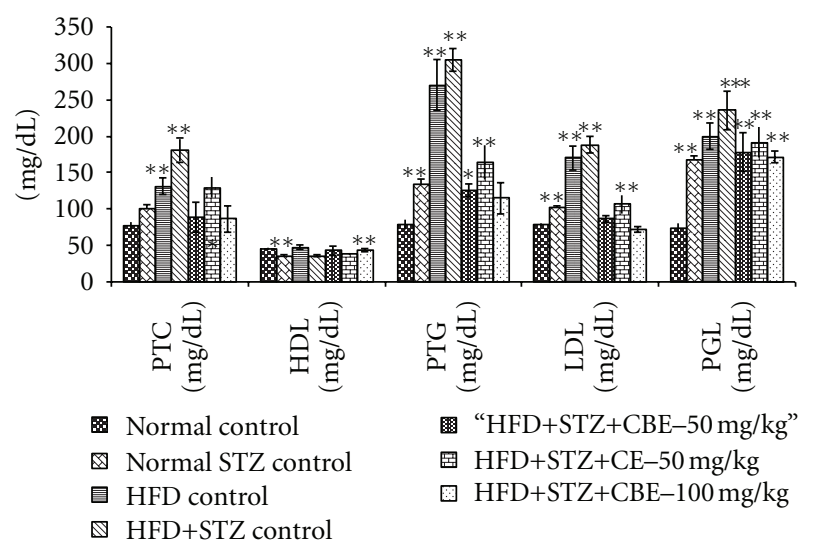

FIGURE 1: Effect of CPQ on biochemical parameters in high-fat diet and low-dose streptozotocin-induced diabetic rats. Values are mean \pm S.E.M. $(N=6)$. All the groups were significant $* * * P<0.001$, and ${ }^{* *} P<0.01$ versus control.

high-fat diet and low-dose streptozocin were observed significantly $(P<0.001)$ increased as compared to normal pellet diet streptozocin treated group (Figure 1). In addition, treatment of HFD-/STZ-induced hyperglycemia with CPQ for 28 days in rats significantly reduced PGL (64.84\%), PTG (61.16\%), LDL (53.98\%), and PTC (48.07\%) level as compared to HFD diet diabetic group. Combination consisting of curcumin with piperine and quercetin (CPQ) also increased the downregulated plasma level of HDL at a rate of $15.81 \%$ and in a concentration of $100 \mathrm{mg} / \mathrm{kg}$.

3.2. Effect of Combination Consisting of Curcumin with Piperine and Quercetin (CPQ) on Body Weight. No mortality was observed in any experimental group throughout the period of investigation. As can be seen from (Figure 2), feeding on HFD combined with low-dose streptozocin showed a significant $(P<0.05)$ reduction in the body weight of HFD-fed rats, which was still considerably higher than NPD-fed rats. However, treatment with CPQ produced increase in body weight as compared to curcumin alone treated group, but the body weight gain during the study was not statistically different between HFD and low-dose streptozocin combined with HFD-treated groups.

\subsection{Effect of CPQ on Oral Glucose Intolerance in High-Fat} Diet and Low-Dose Streptozotocin-Induced Diabetic Rats. As can be seen from (Figure 3), feeding the animal with highfat diet and low-dose STZ showed significant elevation in plasma glucose $(P<0.001)$ seen after, just before OGTT at the end of 8 weeks of feeding (Figure 3 ). Oral glucose tolerance test revealed that low-dose streptozocin combined with HFD-fed rats showed significant impairment in glucose tolerance to exogenously administered glucose $(2 \mathrm{~g} / \mathrm{kg})$ as evident from elevated glycemic levels at 30,60, 90, and 120 min after glucose challenge, compared with the vehicletreated normal pellet diet (NPD) control group $(P<0.05)$. Treatment with CPQ consisting of curcumin with piperine and quercetin (50 and $100 \mathrm{mg} / \mathrm{kg}$ p.o) once daily for 4 weeks

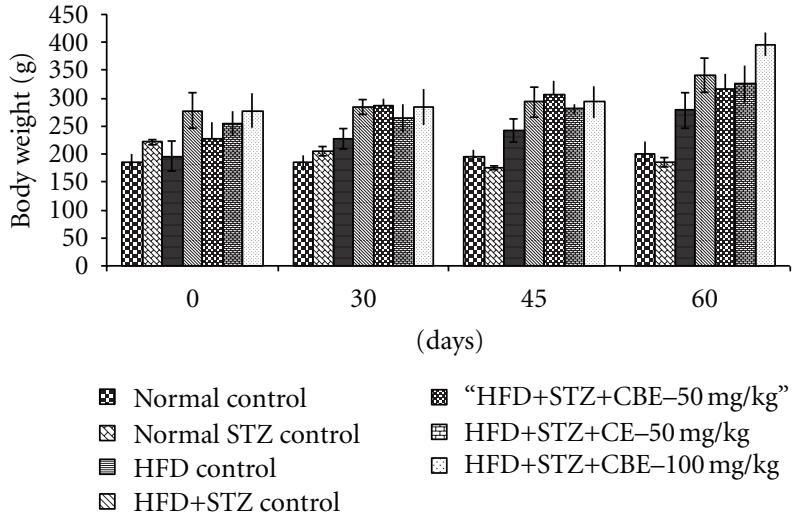

Figure 2: Effect of CPQ on body weight in high-fat diet and lowdose streptozotocin induced diabetic rats. Values are mean \pm S.E.M. $(N=6)$.

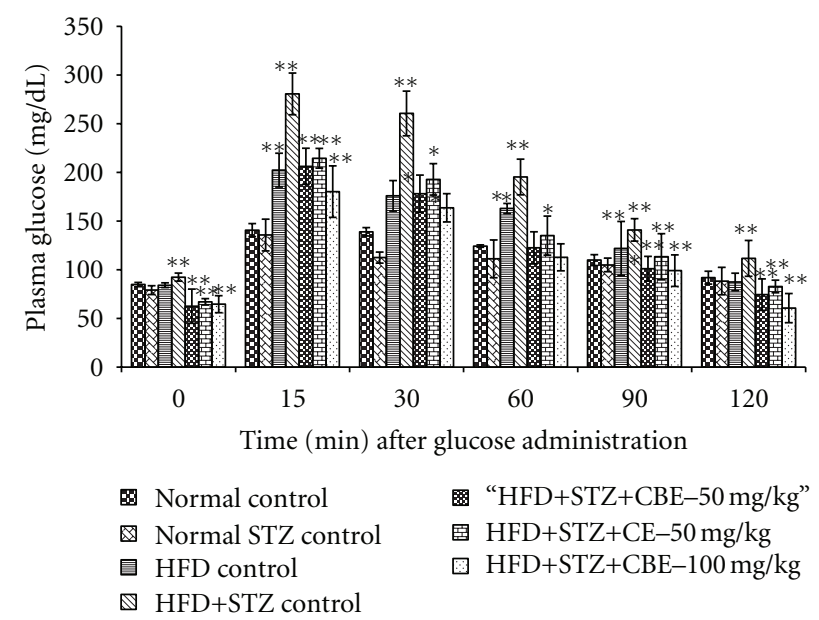

FIGURE 3: Effect of CPQ on oral glucose intolerance in high-fat diet and low-dose streptozotocin-induced diabetic rats. Values are mean \pm S.E.M. $(N=6)$. All the groups were significant ${ }^{* *} P<0.01$, and ${ }^{*} P<0.05$ versus control.

significantly $(P<0.001)$ decreased plasma glucose levels in HFD and low-dose streptozocin-treated diabetic rats. Furthermore, CPQ significantly improved glucose tolerance $(P<0.05)$ to exogenously administered glucose $(2 \mathrm{~g} / \mathrm{kg})$ after 60,90 , and 120 min interval on OGTT in HFD and low-dose streptozocin fed rats compared with the untreated control HFD and low-dose streptozocin fed rats.

3.4. Effect of CPQ on Oxidative Stress Parameters in HighFat Diet and Low-Dose Streptozotocin-Induced Diabetic Rats. Treatment with combination consisting of curcumin with piperine and quercetin $(\mathrm{CPQ})$ showed significant changes in catalase, glutathione, and superoxide dismutase (SOD) levels in diabetic rats. The activity of catalase, GSH, and SOD was decreased significantly $(P<0.01)$ in high-fat diet and low-dose streptozocin as compared to normal control group (Table 1). The decreased levels of antioxidant enzymes in 
TABLE 1: Effect of CPQ extract on oxidative stress parameters in high-fat diet and low-dose streptozotocin-induced diabetic rats.

\begin{tabular}{|c|c|c|c|}
\hline Groups & $\mathrm{SOD}(\mathrm{U} / \mathrm{mL})$ & CAT (U/mL) & GSH-Px ( $\mu$ mole/gHb) \\
\hline Normal control & $8.39 \pm 0.02^{* *}$ & $3.54 \pm 0.03^{* *}$ & $0.00059 \pm 0.001^{* *}$ \\
\hline Normal STZ control & $4.51 \pm 0.03$ & $1.32 \pm 0.13$ & $0.0038 \pm 0.001$ \\
\hline HFD control & $6.62 \pm 0.12^{* *}$ & $2.10 \pm 0.01^{* *}$ & $0.0042 \pm 0.002^{* *}$ \\
\hline HFD + STZ control & $4.38 \pm 0.13$ & $1.08 \pm 0.02^{* *}$ & $0.00283 \pm 0.0017$ \\
\hline CPQ (50 mg/kg/day) & $7.91 \pm 0.03^{* *}$ & $2.80 \pm 0.16^{* *}$ & $0.0048 \pm 0.003^{* *}$ \\
\hline CPQ (100 mg/kg/day) & $9.05 \pm 0.03^{* *}$ & $3.31 \pm 0.01^{* *}$ & $0.005 \pm 0.003^{* *}$ \\
\hline Curcumin $(100 \mathrm{mg} / \mathrm{kg} /$ day $)$ & $6.82 \pm 0.027^{* *}$ & $2.57 \pm 0.02^{* *}$ & $0.0049 \pm 0.001^{* *}$ \\
\hline
\end{tabular}

Values are mean \pm SEM; $N=6 ; * * P<0.01$ as compared to HFD + STZ control.

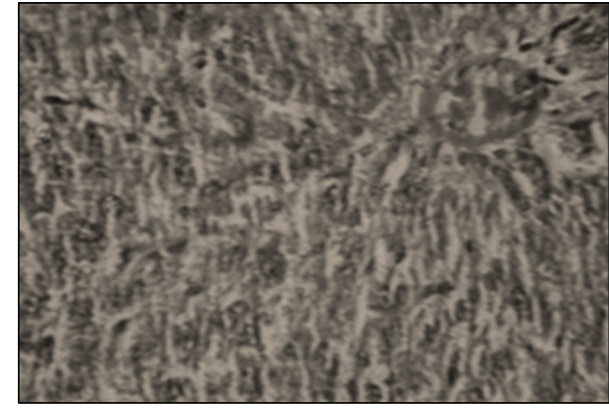

(a)

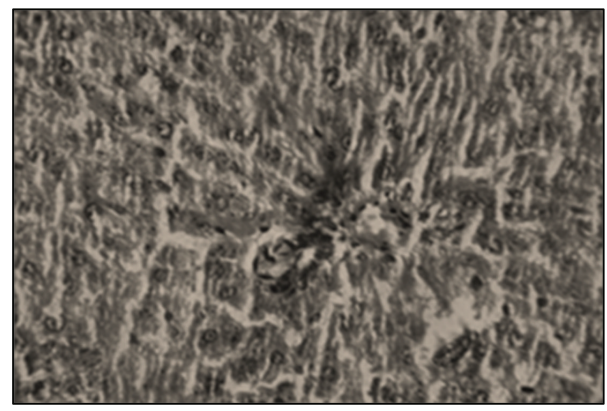

(c)

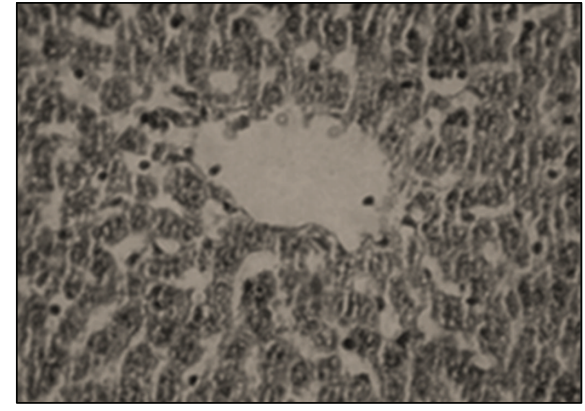

(b)

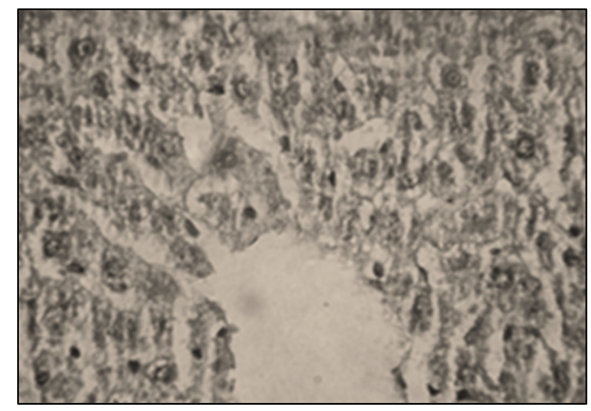

(d)

Figure 4: Histopathological structure of liver. (a) Control group animals; (b) diabetic group animals; (c) treated with CPQ at the dose of $100 \mathrm{mg} / \mathrm{kg}$ of BW; (d) treated with curcumin at the dose of $100 \mathrm{mg} / \mathrm{kg}$ of BW.

diabetes-induced animals, was significantly elevated by the administration of CPQ.

3.5. Histopathological Studies. Animals fed on high-fat diet along with low-dose streptozocin diet appeared healthy and showed no pathological sign of abnormalities during feeding period of 8 weeks. Combination of high-fat diet and 30$\mathrm{mg} / \mathrm{kg}$ body weight STZ produces mild changes in the liver of diabetic rats showed degeneration and scattered necrotic cells, multifocal mild degree per portal lymphocytic infiltration, swollen cytoplasmic hydropic, and microvesicular vacuoles were observed (Figure 4). However, treatment with combination consisting of curcumin with piperine and quercetin (CPQ) at doses of $100 \mathrm{mg} / \mathrm{kg}$ decreased the granular degeneration in diabetic liver. None of the rats treated with high-fat diet and streptozotocin or streptozotocin alone nor any of the untreated controls demonstrated either grossly visible or microscopic renal abnormalities. No grossly visible or microscopic abnormality was seen in the pancreas among all groups of rats used in this study.

\section{Discussion}

It is widely considered that obesity threatens to become the foremost cause of chronic disease in the world. Being obese can induce multiple metabolic abnormalities that contribute to obesity, dyslipidemia, cardiovascular disease, glucose intolerance or type 2 diabetes and other chronic disorders [20]. Despite the plethora of research data available on obesity, it still remains; largely an unsolved medical problem. Diet exercise and nutrition supplementation play a key role in its prevention and treatment. Comparative bioavailability 
studies of curcumin and combination consisting of curcumin with piperine and quercetin (CPQ) were carried out in rats under fed conditions, and the result shows significant increase in serum curcumin concentration on the inclusion of piperine and quercetin in the CPQ. Hence in the present study, we have isolated curcumin, piperine, and quercetin from Curcuma longa Linn, Piper nigrum, and Allium cepa, respectively and tested this combination consisting of curcumin with piperine and quercetin (CPQ) compounds to attenuate metabolic changes associated with high-fat diet and low-dose streptozotocin-induced rats. Acute toxicity study is carried out on laboratory animals by using high dose (sufficient to produce death or morbidity) of the substance in question and based on previous report on its toxicity or toxicity of structurally related compounds. As there was no previous report on toxicity of combination consisting of CPQ, as per OECD guidelines, three dose levels starting at 500, 1000, and limit dose $2000 \mathrm{mg} / \mathrm{kg}$ were selected for acute toxicity study. The animals did not show any changes in the general appearance during the observation period. There was no significant difference in body weight between control and treatment groups. Food and water intake showed daily fluctuations but were within the range of control animals. No mortality was observed in both control and in groups of all selected dose levels.

Therefore, investigators have started to develop a rat model by the combination of high-fat diet and low-dose streptozocin (STZ) that would closely mimic the natural history and metabolic characteristics of type 2 diabetes in humans. Currently, many studies have reported that highfat diet feeding rats develop insulin resistance at first, and then an injection of a low dose of STZ at the same time has been known to induce partial dysfunction of beta cell for suppressing the insulin secretion, which works as a compensation to insulin resistance with the result of persistent hyperglycemia $[21,22]$.

The present study demonstrates that the rats when fed with high-fat diet for 8 weeks combined with low-dose streptozotocin resulted in significant elevation of plasma glucose as evident from elevated glycemic levels at 30, 60, 90 , and $120 \mathrm{~min}$ after glucose challenge, compared with the vehicle-treated normal pellet diet (NPD) control group $(P<$ 0.05 ) and exhibited the characteristic features of obesity and glucose intolerance, namely, increased body weight, hypertriglyceridemia, hypercholesterolemia, and impaired glucose tolerance compared with NPD fed control groups and is in agreement with the findings of Buettner et al., (2000) [23]. In addition, HFD fed rats also showed significant elevation in plasma glucose and impairment in glucose tolerance to exogenously administered glucose as compared with STZ control rats. There are multiple mechanisms involved for the development of insulin resistance and glucose intolerance in high-fat diet fed rats. However, it could be hypothesized that the nature of dietary fatty acid might be involved in altering the cell membrane composition, thereby impeding the binding of insulin to its receptor leading to significant increase in plasma glucose levels in insulin-resistant HFD fed rats when compared with NPD fed normal rats following low dose of STZ (30 $\mathrm{mg} \mathrm{kg}^{-1}$, i.p.).
This is the first study to show that the treatment of the diabetic rats with CPQ resulted in marked decrease in plasma glucose, triglycerides, total cholesterol, and LDL with a concomitant increase in plasma HDL in HFD combined with low dose STZ-induced diabetic rats. In addition, with respect to the in vivo antioxidant enzymes, the pretreatment with combination consisting of "curcumin with piperine and quercetin" (100 mg/kg) once daily for 28 days had a potent increasing effect on serum glutathione and catalase activities, along with elevating SOD activities compared to diabetic group.

The antioxidative property of combinatorial extract certainly is due to its chemical constituents predominantly due to the presence of phenolics which is reported in preliminary phytochemical screening of CPQ. It is further supported in literature that plant phenolics are well known for their antioxidant activity. The phenolics in CPQ may thus play a protective role against the oxidative damage to the pancreatic beta cells. Beta cells are very susceptible to oxidative changes since they possess a low antioxidative capacity $[24,25]$. The results obtained strongly indicate that CPQ consisting of "curcumin with piperine and quercetin" are important sources of natural antioxidants as it significantly $(P<0.01)$ prevented the high-fat diet and low-dose streptozotocininduced changes in the oxidative stress parameters.

Further development of glucose intolerance in HFD combined with low-dose STZ-induced diabetic rats is also in agreement with the data obtained in the literature [26]. The criterion for the predictive diagnosis of diabetic disease is based on the glyco-metabolic features. Here, we investigate an OGTT test to observe the glycemic response to exogenously administered glucose in different groups of rats after 8 weeks of dietary manipulation. The OGTT, defined as the "golden standard", is a method of assessing glucose tolerance and diagnosing diabetes mellitus. The OGTT is advantageous as it is performed under rather physiological conditions, as glucose is given per os thus preserving the effect of entero hormone response and the physiological kinetics of glucose absorption [27]. In this study, the decrease of blood glucose levels at given times after glucose load indicated increased peripheral utilization of glucose by combination consisting of "curcumin with piperine and quercetin". One possible mechanism of these drugs to be effective on postprandial blood glucose is due to inhibition of $\alpha$-glucosidase enzyme. It may also due to an increase in insulin secretion by pancreas [28].

Treatment with CPQ decreased the granular degeneration and alleviated the histopathological changes caused by diabetes in the liver of NIDDM rats. The literature review clearly indicates that fatty infiltration of the aortic subendothelium and deposition of fat in livers were observed in diabetic rats induced by streptozotocin [29]. No special renal abnormalities and pancreatic lesions in relation to any of the high-fat diet could be established which could be due to low dose of streptozocin used in the study. The curative and preventive property of combinatorial extract in diabetic rats may be due to its improvement in glucose tolerance and antioxidant property. 


\section{Conclusion}

In conclusion, the present data supports potent lipid lowering action of combination consisting of "curcumin with piperine and quercetin" in the HFD and low-dose streptozotocin-induced diabetic rats thus further corroborate its potential in the treatment of glucose tolerance associated with excess dietary fat intake, obesity, and type 2 diabetes. Future work needs to be done in the direction of use of CPQ for clinical studies with the aim to elucidate the molecular and cellular mechanism involved with the usage of these nutraceuticals for the prevention of metabolic syndrome.

\section{References}

[1] K. G. M. M. Alberti, P. Zimmet, and J. Shaw, "The metabolic syndrome-a new worldwide definition," The Lancet, vol. 366, no. 9491, pp. 1059-1062, 2005.

[2] K. G. M. M. Alberti, P. Zimmet, and J. Shaw, "Metabolic syndrome - a new world-wide definition. A consensus statement from the International Diabetes Federation," Diabetic Medicine, vol. 23, no. 5, pp. 469-480, 2006.

[3] M. P. Stern, K. Williams, C. González-Villalpando, K. J. Hunt, and S. M. Haffner, "Does the metabolic-syndrome improve identification of individuals at risk of type 2 diabetes and/or cardiovascular disease?" Diabetes Care, vol. 27, no. 11, pp. 2676-2681, 2004.

[4] R. B. Arora, V. Kapoor, N. Basu, and A. P. Jain, "Antiinflammatory studies on Curcuma longa (turmeric)," The Indian Journal of Medical Research, vol. 59, no. 8, pp. 1289 1295, 1971.

[5] Y. Kiso, Y. Suzuki, and N. Watanabe, "Antihepatotoxic principles of Curcuma longa rhizomes," Planta Medica, vol. 49, no. 3, pp. 185-187, 1983.

[6] N. Venkatesan, D. Punithavathi, and V. Arumugam, "Curcumin prevents adriamycin nephrotoxicity in rats," British Journal of Pharmacology, vol. 129, no. 2, pp. 231-234, 2000.

[7] A. J. Ruby, G. Kuttan, K. Dinesh Babu, K. N. Rajasekharan, and R. Kuttan, "Anti-tumour and antioxidant activity of natural curcuminoids," Cancer Letters, vol. 94, no. 1, pp. 79-83, 1995.

[8] K. K. Sharma and S. P. Sharma, "Effect of onion on blood cholesterol, fibrinogen and fibrinolytic activity in normal subjects," Indian Journal of Pharmacology, vol. 8, pp. 231-233, 1976.

[9] Y. Sugiyama, S. Kawakishi, and T. Osawa, "Involvement of the $\beta$-diketone moiety in the antioxidative mechanism of tetrahydrocurcumin," Biochemical Pharmacology, vol. 52, no. 4, pp. 519-525, 1996.

[10] W. C. Jordan and C. R. Drew, "Curcumin—a natural herb with anti-HIV activity," Journal of the National Medical Association, vol. 88, no. 6, p. 333, 1996.

[11] R. Srivastava, M. Dikshit, R. C. Srimal, and B. N. Dhawan, "Anti-thrombotic effect of curcumin," Thrombosis Research, vol. 40, no. 3, pp. 413-417, 1985.

[12] R. Kuttan, P. Bhanumathy, K. Nirmala, and M. C. George, "Potential anticancer activity of turmeric (Curcuma longa)," Cancer Letters, vol. 29, no. 2, pp. 197-202, 1985.

[13] R. Srivastava, M. Dikshit, R. C. Srimal, and B. N. Dhawan, "Anti-thromboticeffect of curcumin," Thrombosis Research, vol. 40, no. 3, pp. 413-417, 1985.
[14] G. Shoba, D. Joy, T. Joseph, M. Majeed, R. Rajendran, and P. S. S. R. Srinivas, "Influence of piperine on the pharmacokinetics of curcumin in animals and human volunteers," Planta Medica, vol. 64, no. 4, pp. 353-356, 1998.

[15] OECD, "Acute oral toxicity test method," in OECD Guidelines for Testing of Chemicals, No. 423. Organization for Economic Cooperation and Development, Paris, France, 2001.

[16] S. Bonner-Weir, "Morphological evidence for pancreatic polarity of $\beta$-cell within islets of Langerhans," Diabetes, vol. 37, no. 5, pp. 616-621, 1988.

[17] H. Aebi, "Catalase," in Methods of Enzymatic Analysis, vol. 2, pp. 673-684, Academic Press, New York, NY, USA, 1974.

[18] E. Beutler, O. Duron, and B. M. Kelly, "Improved method for the determination of blood glutathione," The Journal of Laboratory and Clinical Medicine, vol. 61, pp. 882-888, 1963.

[19] S. L. Marklund, "Properties of extracellular superoxide dismutase from human lung," Biochemical Journal, vol. 220, no. 1, pp. 269-272, 1984.

[20] S. Wild, G. Roglic, A. Green, R. Sicree, and H. King, "Global prevalence of diabetes: estimates for the year 2000 and projections for 2030," Diabetes Care, vol. 27, no. 5, pp. 10471053, 2004.

[21] K. Srinivasan, B. Viswanad, L. Asrat, C. L. Kaul, and P. Ramarao, "Combination of high-fat diet-fed and low-dose streptozotocin-treated rat: a model for type 2 diabetes and pharmacological screening," Pharmacological Research, vol. 52, no. 4, pp. 313-320, 2005.

[22] M. J. Reed, K. Meszaros, L. J. Entes et al., "A new rat model of type 2 diabetes: the fat-fed, streptozotocin-treated rat," Metabolism: Clinical and Experimental, vol. 49, no. 11, pp. 1390-1394, 2000.

[23] R. Buettner, C. B. Newgard, C. J. Rhodes, and R. M. O'Doherty, "Correction of diet-induced hyperglycemia, hyperinsulinemia, and skeletal muscle insulin resistance by moderate hyperleptinemia," American Journal of Physiology, vol. 278, no. 3, pp. E563-E569, 2000.

[24] M. Hotta, F. Tashiro, H. Ikegami et al., "Pancreatic $\beta$ cellspecific expression of thioredoxin, an antioxidative and antiapoptotic protein, prevents autoimmune and streptozotocininduced diabetes," Journal of Experimental Medicine, vol. 188, no. 8, pp. 1445-1451, 1998.

[25] Y. Kajimoto and H. Kaneto, "Role of oxidative stress in pancreatic $\beta$-cell dysfunction," Annals of the New York Academy of Sciences, vol. 1011, pp. 168-176, 2004.

[26] C. Ito, R. Maeda, S. Ishida, H. Harada, N. Inoue, and H. Sasaki, "Importance of OGTT for diagnosing diabetes mellitus based on prevalence and incidence of retinopathy," Diabetes Research and Clinical Practice, vol. 49, no. 2-3, pp. 181-186, 2000.

[27] F. Belfiore and S. Iannello, "Insulin resistance in obesity: metabolic mechanisms and measurement methods," Molecular Genetics and Metabolism, vol. 65, no. 2, pp. 121-128, 1998.

[28] B. K. Chakravarthy, S. Gupta, S. S. Gambhir, and K. D. Gode, "Pancreatic beta-cell regeneration in rats by (-)-epicatechin," The Lancet, vol. 2, no. 8249, pp. 759-760, 1981.

[29] S. K. Mitra, S. Gopumadhavan, T. S. Muralidhar, and S. J. Seshadri, "Effect of D-400, a herbomineral formulation on liver glycogen content and microscopic structure of pancreas and liver in streptozotocin induced diabetes in rats," Indian Journal of Experimental Biology, vol. 34, no. 10, pp. 964-967, 1996. 

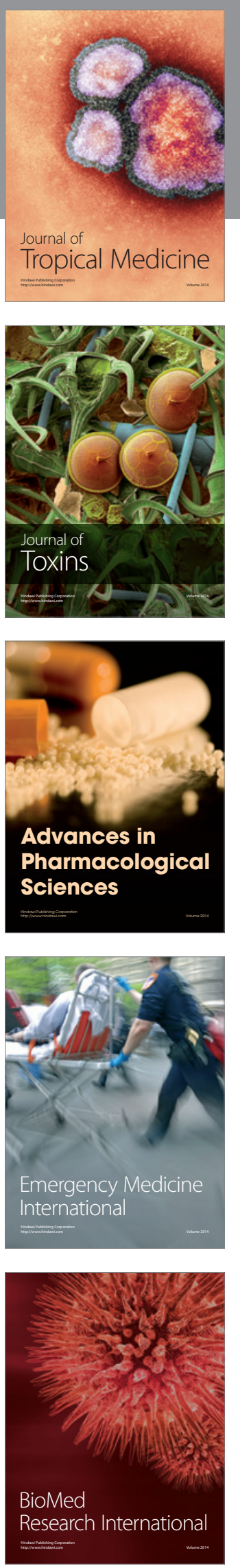
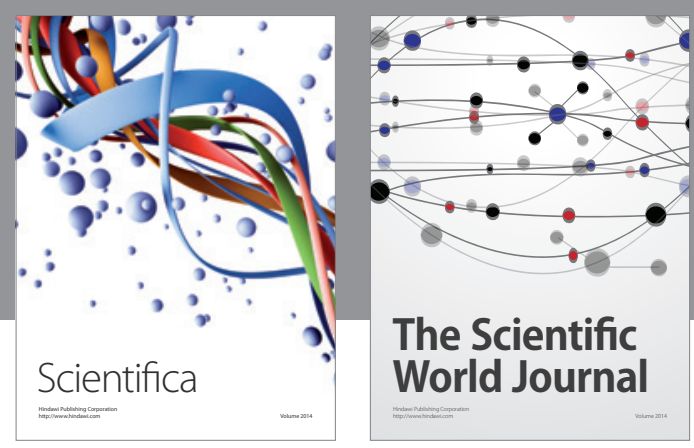

The Scientific World Journal
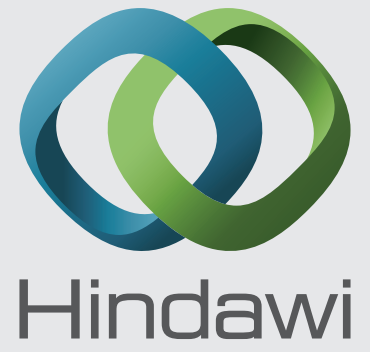

Submit your manuscripts at

http://www.hindawi.com
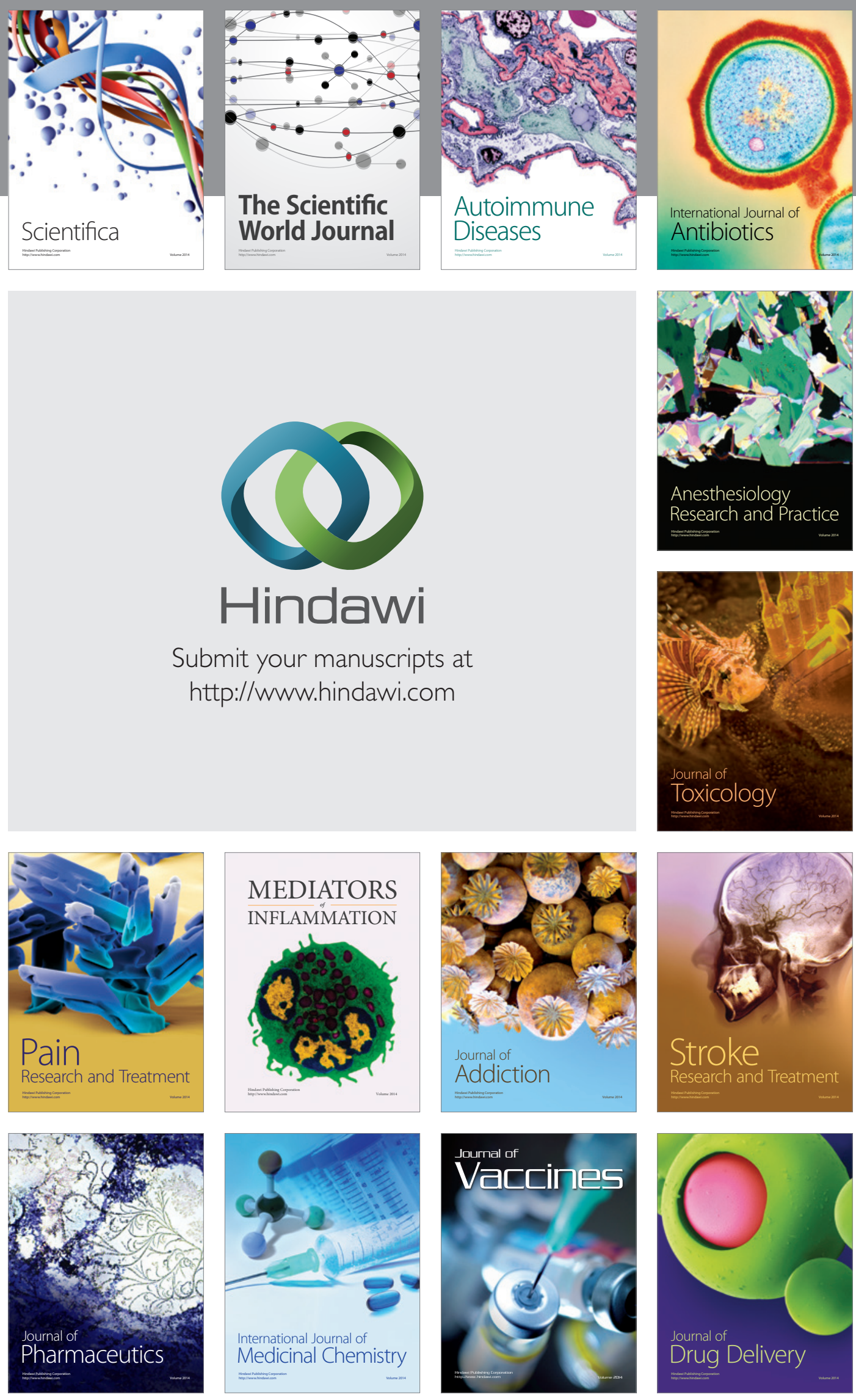